\title{
Optimum stem length for mitigation of periprosthetic fracture risk following primary total knee arthroplasty: a finite element study
}

\author{
Noel Conlisk ${ }^{1,2,5}$ • Colin R. Howie ${ }^{1,4} \cdot$ Pankaj Pankaj ${ }^{1,3,5}$
}

Received: 18 July 2016 / Accepted: 24 October 2016/ Published online: 3 November 2016

(c) The Author(s) 2016. This article is published with open access at Springerlink.com

\begin{abstract}
Purpose Due to age-related changes to the material properties and thinning of the cortical bone structure, older patients with osteoporosis may be at greater risk of femoral fracture following total knee arthroplasty. This study investigates whether there is a potential role for stemmed prostheses in such scenarios to help mitigate peri-implant fracture risk, and if so what should the optimum stem length be to balance surgical bone loss with reduced fracture risk. Methods Finite element models of the distal femur implanted with four different implant types: a posterior stabilising implant, a total stabilising implant with short stem $(12 \mathrm{~mm} \times 50 \mathrm{~mm})$, a TS implant with medium stem $(12 \mathrm{~mm} \times 75 \mathrm{~mm})$, and a TS implant with long stem $(12 \mathrm{~mm} \times 100 \mathrm{~mm})$, were developed and analysed in this study. Osteoporotic properties were applied to the implanted femurs and the periprosthetic stresses and strains of each were recorded.
\end{abstract}

Noel Conlisk

noel.conlisk@ed.ac.uk

1 Orthopaedic Engineering, The University of Edinburgh, Edinburgh, UK

2 School of Clinical Sciences, The University of Edinburgh, Edinburgh, UK

3 School of Engineering, The University of Edinburgh, Edinburgh, UK

4 Department of Orthopaedics, New Royal Infirmary of Edinburgh, Old Dalkeith Road, Little France, Edinburgh, UK

5 Institute for Bioengineering, The University of Edinburgh, Faraday Building, The King's Buildings, Edinburgh EH9 3JL, UK
Results All stem lengths examined were found to lead to a reduction in periprosthetic stress in comparison with a primary stemless implant, with short-, medium-, and longstemmed implants leading to an 11,26 , and $29 \%$ reduction in stress, respectively.

Conclusion The results of this study show that periprosthetic stress and therefore fracture risk in old osteoporotic patients may be reduced through the use of stemmed femoral components. Of the three stems investigated, a mediumlength stem is found to represent the best balance between bone preservation at the time of surgery and reduction in periprosthetic stress following implantation.

Keywords Optimum stem length · Distal femur · Total knee arthroplasty $\cdot$ Finite element $\cdot$ Periprosthetic stress

\section{Introduction}

A rare but potentially devastating failure mode of primary total knee arthroplasty (TKA) is periprosthetic fracture. This mode of failure can arise due to a number of different factors such as: a direct trauma to the replaced joint resulting from a low-velocity fall or car accident [26], loss of supporting bone [29, 33] due to stress shielding, osteolysis and osteoporosis, and an increase in localised stress concentrations due to loosening [21]. Older patients with osteoporosis may be particularly at risk of femoral fracture following TKA, due to alterations in the material properties of the bone as a result of ageing, and thinning of the cortical bone structure through endosteal trabecularisation $[6,28]$. The current incidence of periprosthetic fracture worldwide following primary TKA is believed to be in the range of $0.6-3.0 \%$ [21, 26, 32]. However, this failure mode has the potential of becoming a more serious clinical issue 
as the population ages and a greater proportion of younger more active patients undergo TKA.

Traditionally, stemmed femoral prostheses are used for revision of failed primary TKAs. In such scenarios, the stem helps to align the prosthesis, aid implant stability in the presence of bone loss [24] and protect bone grafts prior to integration with the host bone through load sharing at the interface [10]. However, the initial stability afforded by the use of large-diameter diaphyseal engaging stem configurations may lead to an increase in bone loss over time as a result of greater levels of stress shielding distally [9, 38]. Studies on cemented non-diaphyseal engaging stems report a slightly more favourable outcome in terms of the level of reported stress shielding $[9,38]$, in comparison with large canal filling stems.

Careful consideration of the impact stemmed prosthesis can have on the mechanical environment of the surrounding bone is essential to a successful patient outcome when treatment requires stemmed prosthesis. By understanding the potential limitations of stems and their impact on the host environment, surgeons and engineers can leverage these devices to generate better clinical outcomes. In a recent in vitro study by Completo et al. [7], the authors suggested the use of a long press-fit stem as a means of reducing strain and therefore fracture risk at the notch edge following notching of the anterior femoral cortex during TKA, thus turning a normal disadvantage of stemmed prosthesis into a clear advantage for a particular clinically encountered scenario.

The present study investigates the application of femoral prostheses with cemented non-diaphyseal engaging stems to determine what influence stemmed implants have on the levels of stress in the region immediately above the implant, for a simulated old patient with osteoporosis, in comparison with primary stemless implants for the same patient. A secondary goal was to determine whether there was an optimum stem length to balance bone loss at the time of surgery with peri-implant stress reduction.

The main hypothesis of the study is that stemmed implants used in a primary setting may be beneficial in reducing periprosthetic stress and therefore mitigating some of the fracture risk associated with ageing and osteoporosis in older patients.

\section{Materials and methods}

\section{Geometry}

The femoral geometry used in this study was a threedimensional virtual reconstruction [3] of the large left fourth-generation composite femur (Sawbones; Pacific Research Laboratories, Vashon, Washington, USA). This

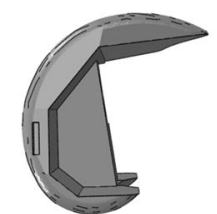

(a)

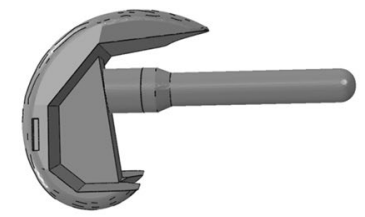

(c)

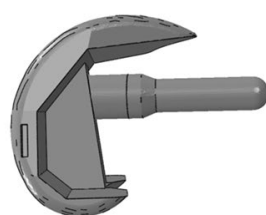

(b)

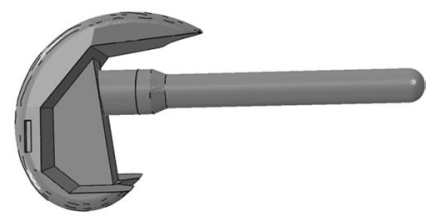

(d)
Fig. 1 Image of all four implant types investigated in this study, a a posterior stabilising (PS) implant, $\mathbf{b}$ a total stabilising implant (TS) with short stem $(12 \mathrm{~mm} \times 50 \mathrm{~mm})$, c a TS implant with medium stem $(12 \mathrm{~mm} \times 75 \mathrm{~mm})$ and $\mathbf{d}$ a TS implant with long stem $(12 \mathrm{~mm} \times 100 \mathrm{~mm})$

geometry was subsequently modified to accept a posterior stabilising (PS) implant, and a total stabilising implant (TS) with femoral stems of varying length. All implants were from the Triathlon ${ }^{\circledR}$ series product line (Stryker ${ }^{\circledR}$, Newbury, United Kingdom). Physical implant measurements and surgical theatre templates were used in conjunction with computer-aided design software (Autodesk Inventor 2010, Autodesk Inc. San Rafael, California, US) to develop 3D models of the femoral implant; the same software was also used to incorporate surgical cuts into the femur to accommodate each implant.

In this study, three different stem lengths were considered for the TS implanted femurs: a short stem $(12 \mathrm{~mm} \times 50 \mathrm{~mm})$, a medium stem $(12 \mathrm{~mm} \times 75 \mathrm{~mm})$ and a long stem $(12 \mathrm{~mm} \times 100 \mathrm{~mm})$. In the Triathlon ${ }^{\circledR}$ product line, stem diameters range in size from $9 \mathrm{~mm}$ to $21 \mathrm{~mm}$ (Stryker ${ }^{\circledR}$, UK); in the present study, a non-canal filling stem diameter of $12 \mathrm{~mm}$ was chosen, as this represents a common size employed by surgeons for cementing.

The four implant configurations investigated in this study are presented in Fig. 1.

\section{Cement layer}

For simplicity, the cement layer was only modelled explicitly from the back surface of the implant box along the stem to a distance of $20 \mathrm{~mm}$ past the stem tip, representing where the cement is retained by the cement restrictor in the clinical setting [16]. The cement layer then extended outward to the endosteal surface of the cortical bone modelling permeation of the cement through the cancellous structure to the cortex, as is achieved in vivo through pressurisation 
Fig. 2 Semi-transparent rendering of a a femur implanted with $50-\mathrm{mm}$ stem, b a femur implanted with 75-mm stem and c a femur implanted with $100-$ $\mathrm{mm}$ stem, with cement, bone and prosthesis regions indicated through the colour coded legend at the bottom (a)

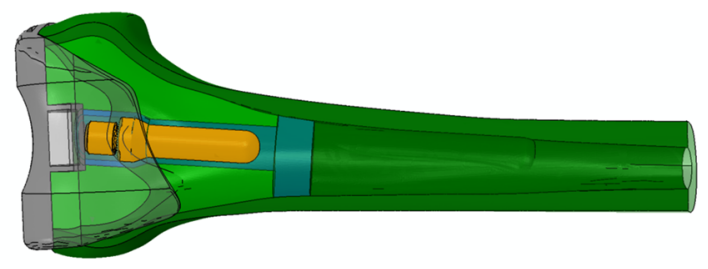

(b)

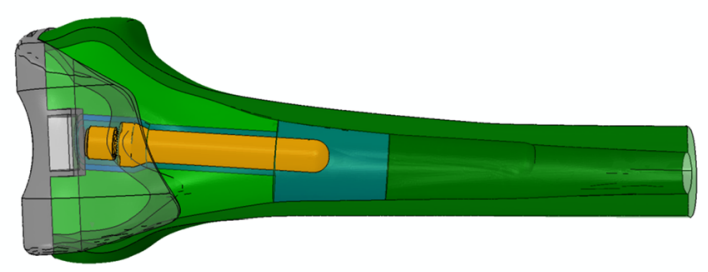

(c)

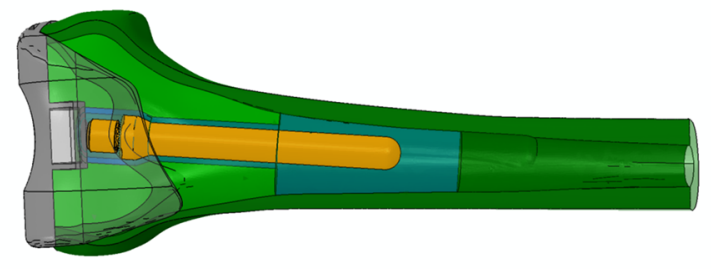

Implant

Stem

Cement layer

Cortical bone

Cancellous bone of the cement into the bone with a cement gun. An assembled view of the cemented stemmed implants is presented in Fig. 2, with implant, stem and bone structures identified.

\section{Interface conditions}

All interfaces were assumed to be fully bonded; the implant-stem assembly was fully tied to the internal surface of the cement, and the external surface of the cement layer tied to the surrounding bone. No relative motion was allowed between the structures modelling full cemented fixation of the implant assembly into the bone.

\section{Material properties}

The femoral component, femoral stem, cement layer and cancellous bone regions were assumed to behave in a linear elastic, isotropic and homogeneous manner. The cortical bone structure, on the other hand, was modelled as inhomogeneous with endosteal thinning of the cortex, using the methodology introduced by Conlisk et al. [13], to simulate an old osteoporotic patient.

In brief, this method involves creating a distribution of Young's modulus which varies linearly from endosteum to periosteum, based on previously reported variations [14], and then offsetting the location of the endosteal Young's modulus by $50 \%$, in line with population values $[6,28]$, to
Table 1 Material properties applied to finite element model

\begin{tabular}{lcl}
\hline Component & $\begin{array}{c}\text { Young's modulus } \\
E\left(\mathrm{~N} / \mathrm{mm}^{2}\right)\end{array}$ & $\begin{array}{l}\text { Poisson's } \\
\text { ratio }(v)\end{array}$ \\
\hline $\begin{array}{l}\text { Cancellous bone } \\
\text { Cortical bone } \\
(\text { endosteum) }\end{array}$ & 155 & 0.3 \\
$\begin{array}{l}\text { Cortical bone } \\
\text { (periosteum) }\end{array}$ & 7000 & 0.3 \\
$\begin{array}{l}\text { Cement } \\
\text { Femoral component } \\
\quad(\text { Co-Cr) }\end{array}$ & 16,700 & 0.3 \\
$\begin{array}{l}\text { Femoral stem } \\
(\mathrm{ti}-6 \mathrm{al}-4 \mathrm{v})\end{array}$ & 210,000 & 0.3 \\
& 110,000 & 0.3 \\
& & 0.3
\end{tabular}

simulate trabecularisation of the cortical bone. The values of Young's moduli and Poisson's ratio applied to all structures in this study are presented in Table 1.

\section{Loading and boundary conditions}

A single flexion angle $\left(48^{\circ}\right)$, representative of maximum load bearing, during the stance phase of gait for a normal walking cycle was investigated in this study and modelled as a static load step. This flexion angle was select for investigation, as previous work has shown maximum periprosthetic stresses and strains to coincide with maximum load 
Fig. 3 a Arrangement of forces at the distal femur, and $\mathbf{b}$ contact areas over which they are applied for $48^{\circ}$ flexion

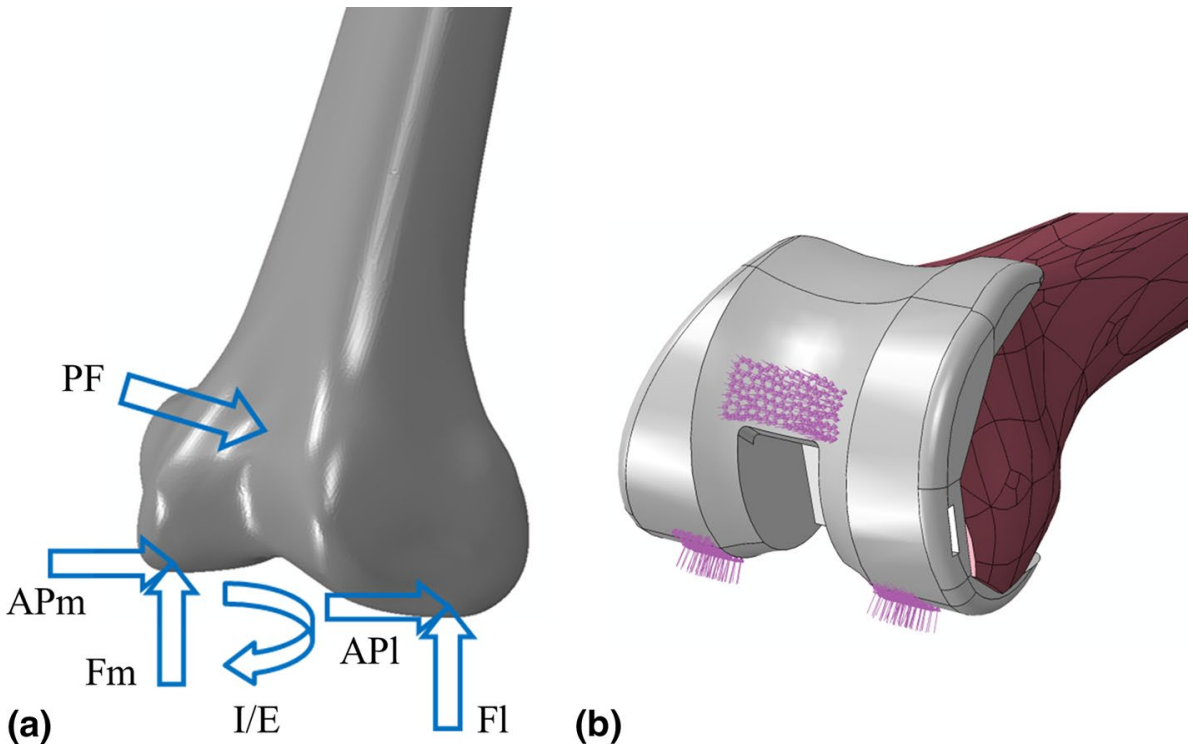

(2\%) change in calculated displacements/stresses, while dramatically increasing simulation runtime. Final FE meshes typically comprised of $>290,000$ quadratic tetrahedral elements (C3D10 M). Simulation runtime for each model was in the region of $2.5 \mathrm{~h}$ on a dual core Intel i5 laptop with $8 \mathrm{~GB}$ of RAM.

\section{Comparative analysis}

A number of transverse sections through each femur (Fig. 4a) were taken to better understand the impact of cemented stems on its mechanical environment and to examine how alterations to the stresses and strains distally may affect other regions of the femur (e.g. the region surrounding the end of the stem). The section $\mathrm{c}-\mathrm{c}$ represents a location just above the implant. Section $d-d$ is taken at the location near the end of the $50-\mathrm{mm}$ stem, section e-e at a location near the end of the 75-mm stem and finally section $\mathrm{f}-\mathrm{f}$ at a location near the end of the $100-\mathrm{mm}$ stem. It should be noted that to ensure consistency of results, all transverse sections were examined for each case.

\section{Results}

It can be seen from Fig. $4 b$ that the majority of the stress (von Mises) is transmitted through the stem-cement construct leading to a reduction in stress distally for femurs implanted with stemmed prostheses (section $\mathrm{c}-\mathrm{c}$ ). As the end of each stem is approached, section $\mathrm{d}-\mathrm{d}$ for the TS implant with 50-mm stem, section e-e for the 75-mm stem and section $\mathrm{f}-\mathrm{f}$ for the $100-\mathrm{mm}$ stem, it can be seen that the stress in the cement increases, indicating increased load transfer at the stem-cement and cement-bone interfaces, reduction in element edge length produced a negligible 
Fig. 4 Showing a the location of each of the transverse sections through the femur, $\mathbf{b}$ the resulting plots of von Mises stress and $\mathbf{c}$ equivalent strain at the transverse sections for all cases investigated (a)

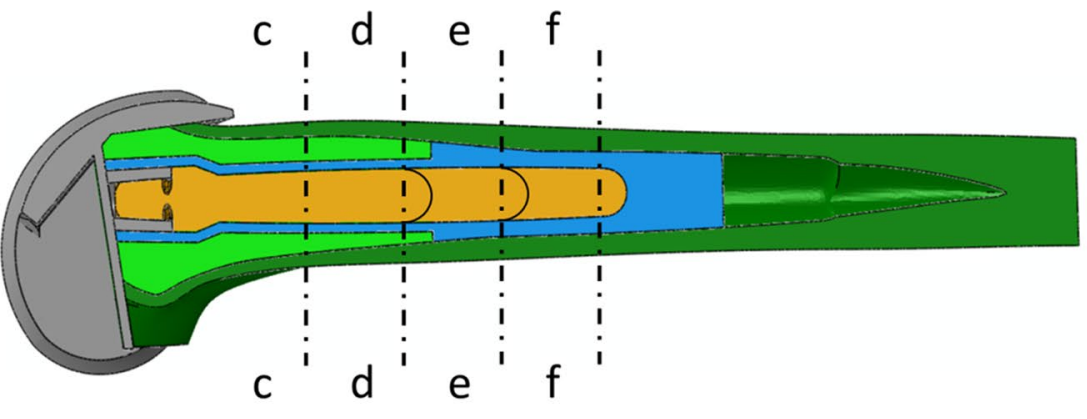

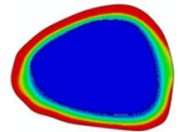
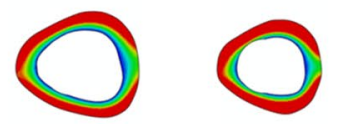

PS
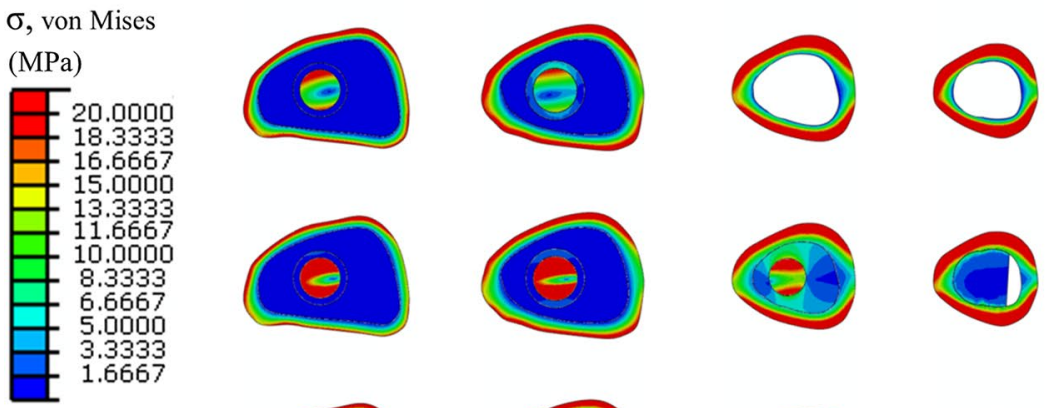

TS $(50 \mathrm{~mm})$
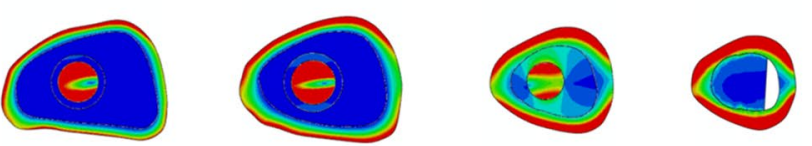

TS (75mm)
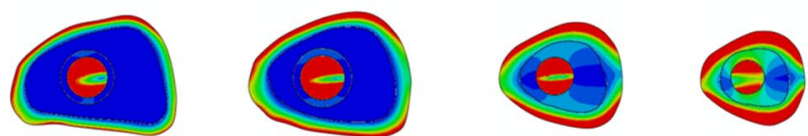

TS $(100 \mathrm{~mm})$

(b)

$\mathrm{c}-\mathrm{C}$

d-d

e-e

$f-f$
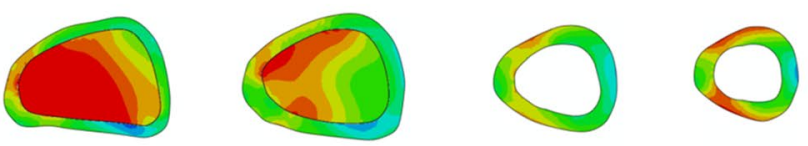

PS
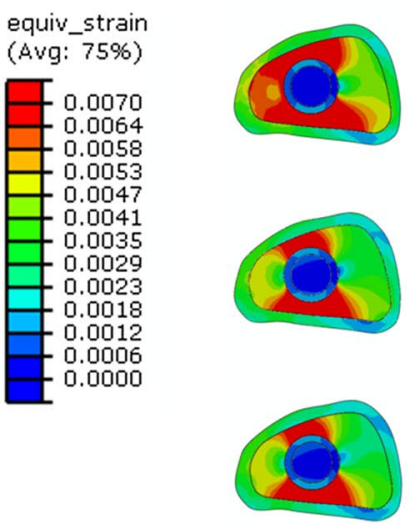

(c)

$\mathrm{c}-\mathrm{c}$
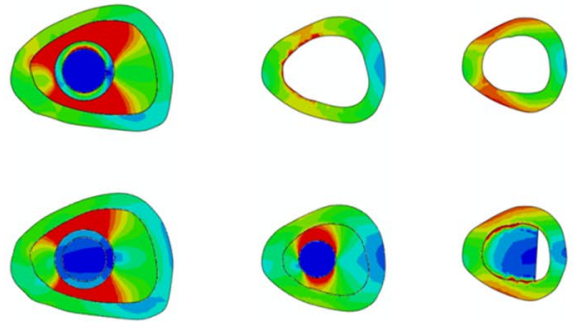

TS $(50 \mathrm{~mm})$

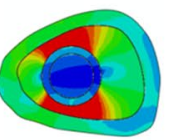

d-d
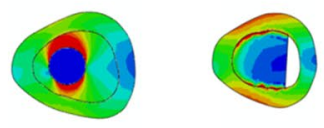

TS $(75 \mathrm{~mm})$

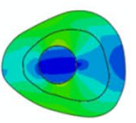

e-e

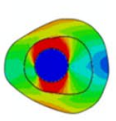

TS $(100 \mathrm{~mm})$

To characterise the influence of stemmed femoral pros-

leading to a slight increase in cortical regions above the end of the stem. Similarly in Fig. 4c, it can be observed from the plots of equivalent strain that the added stiffness of the stems serve to reduce the level of strain in the distal femur. thesis on the periprosthetic mechanical environment in a more quantifiable manner, the values of von Mises stress and equivalent strain were recorded at four cortical points 


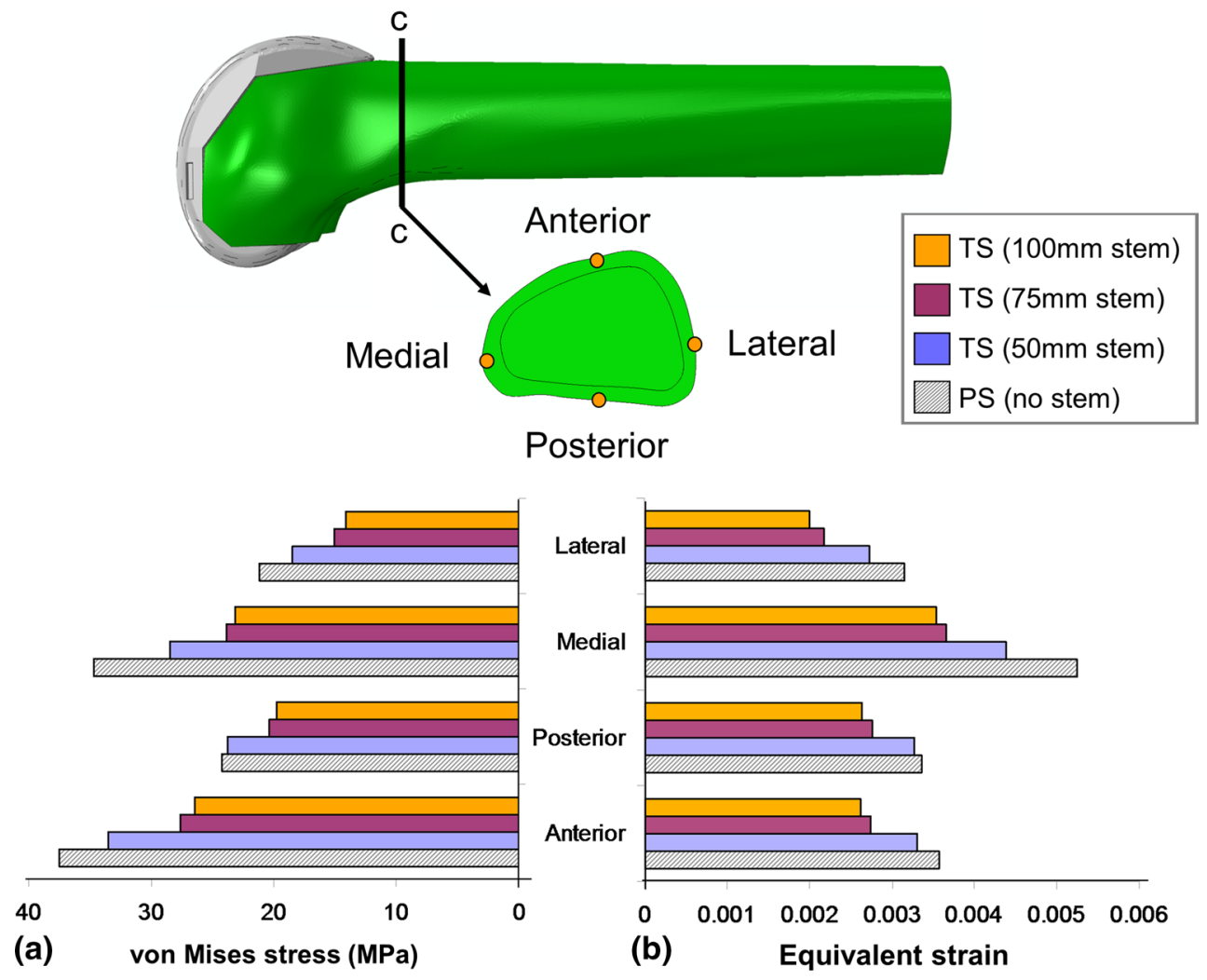

Fig. 5 a von Mises stress and $\mathbf{b}$ equivalent strain at each of the points of interest for varying stem length

Table 3 Overall percentage reduction in periprosthetic stress and strain for femurs implanted with stemmed implants relative to a PS implanted femur

\begin{tabular}{llcc}
\hline Model & Stem length $(\mathrm{mm})$ & Decrease in von Mises stress $(\%)$ & Decrease in equivalent strain $(\%)$ \\
\hline PS & N/A & 0.0 & 0.0 \\
TS short & 50 & 10.9 & 10.0 \\
TS medium & 75 & 25.7 & 25.5 \\
TS long & 100 & 28.7 & 29.3 \\
\hline
\end{tabular}

Where for each femur, values of stress and strain are calculated based on the average of the four cortical points of interest of interest, at the location of the transverse section c-c, as shown in Fig. 5a, b, respectively. It can be seen for all stemmed femoral components that the largest values of strains occur on the medial cortex and the largest reported values of stress act on the anterior femoral cortex. The short stem $(50 \mathrm{~mm})$ is observed to have a response closest to that of the stemless PS implanted femur and leads to the highest levels of periprosthetic stress of all the revision stemmed implants investigated.

If we compare the average values of stress and strain from all stemmed prostheses to that of the PS implanted femur, the percentage reduction in stress and strain due to stem length can be determined (Table 3 ). The table shows that on average the $50-\mathrm{mm}$ stem resulted in an overall decrease of approximately $11 \%$ in periprosthetic stress and $10 \%$ in periprosthetic strain, the $75-\mathrm{mm}$ stem lead to a $26 \%$ reduction in both stress and strain, whereas the $100-\mathrm{mm}$ stem resulted in a $29 \%$ reduction in stress and strain.

\section{Discussion}

The most important finding of this study was that cemented non-diaphyseal engaging stems were beneficial in reducing periprosthetic stress. The level of reduction was observed to be related to the length of the stem employed, with a medium-length stem representing the best balance between bone preservation at the time of surgery and reduction in periprosthetic stress following implantation. 
Prosthesis-induced stress shielding is frequently observed following TKA [1, 25, 31, 33, 36, 37]. However, it is important to recognise that internal stresses need to be in equilibrium with externally applied forces; as a result, a reduction in stress in some regions will lead to an increase in stresses in others [31]. In the context of the current study, the observed reduction distally leads to an increase in stress proximally in the cement and stem structures. However, it should be noted that as the load transfer is distributed along the length of the stem-cement construct, the overall result is that implanted femurs with longer stems (i.e. 75 and $100 \mathrm{~mm}$ stems) are subject to slightly lower stresses and strains proximally than the stemless or short-stemmed cases. Furthermore, as the cortex is significantly thicker in the mid-shaft, it may be less susceptible to fracture than the much thinner and less stiff cortical bone structure found immediately adjacent to the implant.

Stems are widely accepted as being necessary to ensure successful outcomes during revision TKA, particularly in instances of severe bone loss $[15,24]$, where bone grafts are required to replace damaged or diseased host bone. In this capacity stems serve to transmit the load to healthy bone structures above the implant and away from the graft, thereby allowing it to fully incorporate into the host structure without risk of damage and overloading [15]. Stemmed prostheses have also found use in the treatment of ligament laxity through aiding in the stability of more constrained implant types [18]. It has also been suggested previously that the addition of a stem may also serve to decrease the likelihood of periprosthetic femoral fracture following notching of the anterior femoral cortex [7, 19].

In the present study, the novel application of stems as a preventative measure against periprosthetic fracture in older patients with osteoporosis was investigated. It was found that the addition of a cemented stem to the distal femur caused a reduction in periprosthetic stress. The short cemented stem $(50 \mathrm{~mm})$ caused a reduction of approximately $11 \%$, while longer stems $(75$ and $100 \mathrm{~mm})$ were seen to result in reductions of 26-29\% when compared with a stemless PS implanted femur. The reduction in strain was observed to follow a similar trend with respect to stem length. Moreover, the reduction in strain which occurred as a result of the medium stem being found to be of a similar magnitude to that found previously in a study of fracture risk following notching [7]. Based on the findings of the present study, it can be seen that the stem length is an important parameter for the reduction in periprosthetic stress in older osteoporotic patients. Of the three stems investigated, the use of a $75 \mathrm{~mm}$ cemented stem is recommended, as this stem affords a reduction in stress almost comparable to the 100 -mm stem (e.g. less than $4 \%$ difference between the two) while also preserving a greater level of bone stock (e.g. less bone removed during implantation of smaller stems). It is also known that shorter stems in general are easier to fit at the time of operation and are less influenced by the curvature of the femur [24].

Moreover, the selection of a smaller stem has been suggested on the grounds of preserving bone density in the femur post-implantation. In a study by van Lenthe et al. [38] on bone remodelling around revision prosthesis, the influence of two stemmed femoral components (thick and thin) relative to a primary implant was investigated. The findings of van Lenthe et al. suggested that a femoral component with a thin stem performed in a similar manner to the primary stemless implant and had a much more favourable rate of bone loss than a femoral component with a thick stem.

It must be noted that while stems provide many immediate benefits, several clinical reports also suggest a higher incidence of fracture in stemmed versus stemless prosthesis. Meek et al. [21] reported a fracture risk of $0.6 \%$ for primary and $1.7 \%$ for revision knee arthroplasty at 5 years; these values increased to 1.3 and $2.2 \%$, respectively, at 10 years. Meek and colleagues identified female patients over 70 years of age to be most at risk of fracture following knee replacement. A study by Singh et al. [32] spanning a 19-year period reported similar periprosthetic fracture rates following primary $(1.1 \%)$ and revision $(2.5 \%)$ knee arthroplasty. Interestingly, very different conclusions were drawn with respect to at-risk patient groups by these two studies. The somewhat contradictory evidence surrounding periprosthetic femoral fracture following primary and revision TKA indicate that the exact mechanisms at work are quite complex and may warrant closer examination. However, it is important to recognise that the implantation of a revision femoral prosthesis in the setting of primary TKA is not the same as implantation of the same prosthesis during revision surgery. Both scenarios will have very different initial conditions, particularly with respect to bone quality for fixation of the prosthesis. It must therefore be considered that the clinical observances that revision stemmed prostheses have a higher fracture rate than primary stemless implants, may not be directly applicable to the present study, as a wide array of factors may influence the outcome in revision surgery (e.g. age, gender, and health). Indeed, a recent retrospective study by Barlow et al. [2] highlighted comparable short-term outcomes (at 49 months) for stemmed and conventional TKA when used in a primary setting.

The present study has some limitations. In this study, only a single diameter of stem was considered, with stem length varied using three common sizes. However, most manufacturers provide a range of different stem diameters, sizes, lengths and end designs, as such alterations to any one of these parameters may also influence the load sharing at the bone-prosthesis interface. It is therefore recommended that further studies be conducted to assess 
the importance of other stem parameters. Another consideration is that only cemented stems were investigated. Cementing in all cases was modelled on a fully bonded metaphysis and pressurising of cement along the stem out to the cortex to achieve optimum load transfer, which may not be fully representative of in vivo conditions. If full contact was not achieved, then this could severely reduce the load sharing capacity of the construct [38] and limit its effectiveness for preventing periprosthetic fracture. Furthermore, a reduction in load sharing may lead to bone loss, and eventual loosening due to overloading of the surrounding cancellous bone [34]. Should loosening occur distally, the accompanied reduction in load sharing could result in the majority of load being transmitted solely through the femoral component-stem assembly, increasing the likelihood of stem junction failure and femoral fracture occurring $[12,17,20,23]$. Periprosthetic stresses resulting from maximum load bearing during walking were examined in this study. Other patterns of gait (e.g. ascending/descending stairs, and squatting) may result in very different distributions of stress in the femur. However, due to the comparative nature of this study, it is unlikely that the conclusions drawn would be significantly affected by alterations to the pattern of loading (once applied uniformly across all model variations). This study examined the initial response of the femur immediately following implantation. It is recognised that bone is dynamic and will remodel in response to the presence of an implant. Therefore, if predicting the long-term survival of the prosthesis is of interest (e.g. loosening or fracture), incorporation of a complex bone remodelling framework, e.g. [27], would be required to adequately capture the response of the femur to disease progression. Finally, only a single femoral geometry with osteoporotic bone properties was considered in this study; future studies should examine whether the observed benefits of cemented stems extend to other femoral geometries and patient types.

Despite these limitations, the findings of this study may aid Surgeons to achieve better post-implantation outcomes for older osteoporotic patients, through an enhanced understanding of how different implant types affect the underlying mechanics of the bone.

\section{Conclusion}

The findings of the present study suggest that a small-diameter medium-length cemented stem could have beneficial applications in reducing periprosthetic stress and therefore fracture risk in elderly patients with osteoporosis. It is recommended that clinical studies should be conducted to confirm the findings of this biomechanical modelling study.
The outcomes of which, in conjunction with the work presented here, could then help to better inform surgical treatment of patients in this particular group.

\section{Compliance with ethical standards}

\section{Conflict of interest None.}

Funding Financial support from the Lothian University Hospitals NHS Trust Brown and Ireland Estates Fund and The University of Edinburgh is gratefully acknowledged, although they had no part in the conception, analysis or reporting of results.

Ethical approval Not required.

\section{Informed consent None.}

Open Access This article is distributed under the terms of the Creative Commons Attribution 4.0 International License (http://creativecommons.org/licenses/by/4.0/), which permits unrestricted use, distribution, and reproduction in any medium, provided you give appropriate credit to the original author(s) and the source, provide a link to the Creative Commons license, and indicate if changes were made.

\section{References}

1. Barink M, Verdonschot N, de Waal Malefijt M (2003) A different fixation of the femoral component in total knee arthroplasty may lead to preservation of femoral bone stock. Proc Inst Mech Eng H 217:325-332

2. Barlow BT, Oi KK, Y-Y Lee, Joseph AD, Alexiades MM (2016) Incidence, indications, outcomes, and survivorship of stems in primary total knee arthroplasty. Knee Surg Sports Traumatol Arthrosc. doi:10.1007/s00167-016-4227-6:1-9

3. Fourth generation femur model, BEL Repository (2008). http://www.biomedtown.org/biomed_town/LHDL/Reception/ datarepository/repositories/BEL/repository/wh_view

4. Bergmann G (2008) Orthoload. Charite-Universitaetsmedizin Berlin. http://www.OrthoLoad.com

5. Bougherara H, Zdero R, Mahboob Z, Dubov A, Shah S, Schemitsch EH (2010) The biomechanics of a validated finite element model of stress shielding in a novel hybrid total knee replacement. Proc Inst Mech Eng H 224:1209-1219

6. Bousson V, Meunier A, Bergot C, Vicaut É, Rocha MA, Morais MH, Laval-Jeantet A-M, Laredo J-D (2001) Distribution of intracortical porosity in human midfemoral cortex by age and gender. J Bone Miner Res 16:1308-1317

7. Completo A, Fonseca F, Relvas C, Ramos A, Simões J (2012) Improved stability with intramedullary stem after anterior femoral notching in total knee arthroplasty. Knee Surg Sports Traumatol Arthrosc 20:487-494

8. Completo A, Fonseca F, Simões JA (2007) Experimental validation of intact and implanted distal femur finite element models. J Biomech 40:2467-2476

9. Completo A, Simões J, Fonseca F (2008) Experimental evaluation of strain shielding in distal femur in revision TKA. Exp Mech 48:817-824 
10. Completo A, Simões JA, Fonseca F (2009) Revision total knee arthroplasty: the influence of femoral stems in load sharing and stability. Knee 16:275-279

11. Conlisk N, Gray H, Pankaj P, Howie CR (2012) The influence of stem length and fixation on initial femoral component stability in revision total knee replacement. Bone Joint Res 1:281-288

12. Conlisk N, Howie CR, Pankaj P (2015) The role of complex clinical scenarios in the failure of modular components following revision total knee arthroplasty: a finite element study. J Orthop Res 33:1134-1141

13. Conlisk N, Howie CR, Pankaj P (2016) An efficient method to capture the impact of total knee replacement on a variety of simulated patient types: a finite element study. Med Eng Phys 38:959-968

14. Donaldson FE, Pankaj P, Cooper DML, Thomas CDL, Clement JG, Simpson AHRW (2011) Relating age and micro-architecture with apparent-level elastic constants: a micro-finite element study of female cortical bone from the anterior femoral midshaft. Proc Inst Mech Eng H 225:585-596

15. Engh GA, Herzwurm PJ, Parks NL (1997) Treatment of major defects of bone with bulk allografts and stemmed components during total knee arthroplasty. J Bone Joint Surg Am Series A 79:1030-1039

16. Faraj AA, Rajasekar K (2006) The effect of two different types of cement restrictors on the femoral cement mantle. Acta Orthop Belg 72:702-708

17. Issack PS, Cottrell JM, Delgado S, Wright TM, Sculco TP, Su EP (2007) Failure at the taper lock of a modular stemmed femoral implant in revision knee arthroplasty-a report of two cases and a retrieval analysis. J Bone Joint Surg Am 89A:2271-2274

18. Kwon O, Kamath A, Voleti P, Garino J (2012) Isolated lateral ligament laxity in primary total knee arthroplasty: cohort study of stemmed versus unstemmed implants. J Bone Joint Surg Br 94-B: 124

19. Lesh ML, Schneider DJ, Deol G, Davis B, Jacobs CR, PelligriniJr VD (2000) The consequences of anterior femoral notching in total knee arthroplasty a biomechanical study. J Bone Joint Surg Am 82:1096

20. Lim L-A, Trousdale RT, Berry DJ, Hanssen AD (2001) Failure of the stem-condyle junction of a modular femoral stem in revision total knee arthroplasty: a report of five cases. J Arthroplasty $16: 128-132$

21. Meek RMD, Norwood T, Smith R, Brenkel IJ, Howie CR (2011) The risk of peri-prosthetic fracture after primary and revision total hip and knee replacement. J Bone Joint Surg $\mathrm{Br}$ 93-B:96-101

22. Moran MF (2005) Computational and experimental assessment of total knee motion. The Pennsylvania State University, State College

23. Nikolopoulos D, Polyzois I, Magnissalis E, Bernard P, Michos I (2012) Fracture at the stem-condylar junction of a modular femoral prosthesis in a varus-valgus constrained total knee arthroplasty. Knee Surg Sports Traumatol Arthrosc 20:1071-1074

24. Patel AR, Barlow B, Ranawat AS (2015) Stem length in revision total knee arthroplasty. Curr Rev Musculoskelet Med 8:407-412

25. Petersen MM, Olsen C, Lauritzen JB, Lund B (1995) Changes in bone mineral density of the distal femur following uncemented total knee arthroplasty. J Arthroplasty 10:7-11

26. Rayan F, Konan S, Haddad FS (2008) A review of periprosthetic fractures around total knee arthroplasties. Curr Orthop 22:52-61

27. Ruimerman R, Hilbers P, van Rietbergen B, Huiskes R (2005) A theoretical framework for strain-related trabecular bone maintenance and adaptation. J Biomech 38:931-941

28. Russo CR, Lauretani F, Seeman E, Bartali B, Bandinelli S, Di Iorio A, Guralnik J, Ferrucci L (2006) Structural adaptations to bone loss in aging men and women. Bone 38:112-118

29. Saari T, Uvehammer J, Carlsson LV, Regnér L, Kärrholm J (2006) Posterior stabilized component increased femoral bone loss after total knee replacement. 5-year follow-up of 47 knees using dual energy X-ray absorptiometry. Knee 13:435-439

30. Shi J (2007) Finite element analysis of total knee replacement considering gait cycle load and malalignment. University of Wolverhampton, Wolverhampton

31. Shi JF, Wang CJ, Laoui T, Hart W, Hall R (2007) A dynamic model of simulating stress distribution in the distal femur after total knee replacement. Proc Inst Mech Eng H 221:903-912

32. Singh JA, Jensen M, Lewallen D (2013) Predictors of periprosthetic fracture after total knee replacement. Acta Orthop 84:170-177

33. Soininvaara TA, Miettinen HJA, Jurvelin JS, Suomalainen OT, Alhava EM, Kröger HPJ (2004) Periprosthetic femoral bone loss after total knee arthroplasty: 1-year follow-up study of 69 patients. Knee 11:297-302

34. Taylor M, Tanner KE (1997) Fatigue failure of cancellous bone: a possible cause of implant migration and loosening. $\mathrm{J}$ Bone Joint Surg Br 79-B:181-182

35. Taylor SJG, Walker PS, Perry JS, Cannon SR, Woledge R (1998) The forces in the distal femur and the knee during walking and other activities measured by telemetry. J Arthroplasty 13:428-437

36. Tissakht M, Ahmed AM, Chan KC (1996) Calculated stressshielding in the distal femur after total knee replacement corresponds to the reported location of bone loss. J Orthop Res 14:778-785

37. van Lenthe GH, de Waal Malefijt MC, Huiskes R (1997) Stress shielding after total knee replacement may cause bone resorption in the distal femur. J Bone Joint Surg Br 79-B:117-122

38. van Lenthe GH, Willems MMM, Verdonschot N, de Waal Malefijt MC, Huiskes R (2002) Stemmed femoral knee prostheses: effects of prosthetic design and fixation on bone loss. Acta Orthop Scand 73:630 